\title{
NFAT transcription factors: from cell cycle to tumor development
}

J.P.B. Viola,

L.D.S. Carvalho,

B.P.F. Fonseca

and L.K. Teixeira
Divisão de Biologia Celular, Instituto Nacional de Câncer,

Rio de Janeiro, RJ, Brasil

\section{Correspondence \\ J.P.B. Viola \\ Divisão de Biologia Celular \\ Instituto Nacional de Câncer (INCA) \\ Rua André Cavalcanti, 37, 5ㅇandar \\ 20231-050 Rio de Janeiro, RJ \\ Brasil \\ Fax: +55-21-3233-1470 \\ E-mail:.jpviola@inca.gov.br \\ Presented at the XI Congresso Brasileiro de Biologia Celular, Campinas, SP, Brazil, July 15-18, 2004.}

Research supported by INCA/FAF, FURNAS Centrais Elétricas S.A.,

and CNPq. L.K. Teixeira and

B.P.F. Fonseca were supported by

an INCA/FAF fellowship.

Received July 16, 2004

Accepted November 12, 2004

\begin{abstract}
The nuclear factor of activated T cells (NFAT) family of transcription factors has been primarily identified in immune cells; however, these proteins have been recently found to be functionally active in several other non-immune cell types. NFAT proteins are activated upon different stimuli that lead to increased intracellular calcium levels. Regardless of their widely known cytokine gene expression properties, NFATs have been shown to regulate other genes related to cell cycle progression, cell differentiation and apoptosis, revealing a broader role for these proteins in normal cell physiology. Several reports have addressed the participation of NFATs in many aspects of malignant cell transformation and tumorigenic processes. In this review, we will discuss the involvement of the different NFAT family members in the regulation of cell cycling, differentiation and tumor formation, and also its implications on oncogenesis. Better understanding the mechanisms by which NFATs regulate cell cycle and tumor-related events should be relevant for the development of rational anti-cancer therapies.
\end{abstract}

Key words

- NFAT

- Cell cycle

- Cancer

- Cell differentiation

- Gene expression

\section{Introduction}

Cell activation is regulated by an interacting network of transcription factors that determine the expression of different genes. It has been shown that several transcription factors, including the nuclear factor of activated T cells (NFAT) family, play an important role in the control of gene expression during cell activation and differentiation. Most of the work on NFAT proteins has been related to immune cell activation and its mediators, such as cytokines (1). However, accumulating evidence has been demonstrat- ing that NFAT transcription factors are present in a wide range of cell types and tissues that are not related to the immune system, and implicated in the regulation of genes that control cell cycle progression, cell development and differentiation, angiogenesis, and possibly tumorigenesis (2-7). Given the importance of these processes in different stages of cell physiology, it is of considerable interest to understand the mechanisms by which NFAT transcription factors affect cell activation and function. Thus, in this review we will focus on the involvement of the NFAT family of transcription factors in cell cycle 
regulation, cell differentiation and oncogenesis.

\section{The NFAT family of transcription factors}

NFAT was first identified in T cells as a rapidly inducible nuclear factor able to bind to the distal antigen receptor responsive element of the human IL-2 promoter (8). Over the past few years, studies from several laboratories have indicated that NFAT proteins regulate the expression of different genes, including signaling proteins, cytokines, cell surface receptors, and cell cycle and apoptosis related proteins. The NFAT family of transcription factors encodes four distinct proteins that are regulated by the calcium/ calcineurin signaling pathway, known as NFAT1 (also called NFATp, NFATc2), NFAT2 (NFATc, NFATc1), NFAT3 (NFATc4), and NFAT4 (NFATx, NFATc3), and another protein named NFAT5 (TonE$\mathrm{BP}$ ), which is regulated by hyperosmotic stress $(1,9)$. NFAT1, the first identified member of the family, was cloned from murine (Ar-5) and human (Jurkat) $\mathrm{T}$ cell cDNA libraries $(10,11)$. A distinct protein, NFAT2, was also cloned from a Jurkat T cell cDNA library (12). cDNA clones encoding two other NFAT proteins, NFAT3 and NFAT4, were isolated from Jurkat $\mathrm{T}$ cell, human peripheral blood lymphocyte and human thymus cDNA libraries $(13,14)$.

Despite their name, NFAT proteins are expressed not only in $\mathrm{T}$ cells, but also in other classes of immune and non-immune cells. NFAT1, NFAT2 and NFAT4 are expressed at the protein level in peripheral $\mathrm{T}$ and $\mathrm{B}$ cells; NFAT1 is also expressed in mast cells, NK cells, and in certain monocytes and macrophages $(1,9,15)$. NFAT1 and NFAT2 mRNAs are expressed in peripheral lymphoid tissues, and NFAT2 mRNA is upregulated in activated T cells and NK cells $(12,14)$. NFAT4 mRNA is expressed at high levels in the thymus and at low levels in peripheral lymphoid tissues $(13,14)$. However, several data have demonstrated that NFAT proteins are also present in different non-related immune cell lines and tissues, such as neuronal cell line and nervous system tissues, endothelial cell line, skeletal and heart muscle, chondrocytes, keratinocytes, and adipocytes among others $(1,5,16)$.

Several isoforms have been described for NFAT1, NFAT2 and NFAT4. Sequence homology represented in all the isoforms suggests two different domains, comprising the DNA-binding domain and the NFAT homology region $(11,17)$. The DNA-binding domain, which is located between amino acid residues 400 and 700, is highly conserved within the NFAT family, and shows moderate sequence similarity to the DNA-binding domains of Rel-family proteins (17). Based on comparisons of binding site sequences, the NFAT binding site is determined to be a 9-bp element, possessing the consensus nucleotide sequence (A/T)GGAAA(A/N)(A/ $\mathrm{T} / \mathrm{C}) \mathrm{N}(1,9)$. In addition, NFAT transcription factors cooperate with AP-1 proteins in DNA binding and transactivation, and this association results in stabilization of the NFAT-DNA interaction $(1,9)$. The NFAT homology region is located in the $\mathrm{N}$-terminal region, comprising 300 amino acids, and shows a strong conservation of several sequence motifs characteristic of the NFAT family $(11,13,14)$.

Further on, NFAT transcription factors have been characterized as cytosolic proteins constitutively expressed in resting cells (1). Upon stimulation of receptors coupled to calcium mobilization, NFAT proteins are activated by calcineurin, the calcium/calmodulinactivated serine-threonine phosphatase $(1,9)$. Activation of NFAT by calcineurin is sensitive to calcineurin inhibitors and immunosuppressive agents, such as cyclosporin A and FK506 $(1,15)$. Three different steps of activation have been defined for NFAT proteins: dephosphorylation, nuclear translocation, and DNA binding (Figure 1). In resting cells, NFAT proteins 
are phosphorylated and cytoplasmic, and show a low affinity for DNA (18). Stimuli that trigger calcium mobilization result in rapid dephosphorylation of NFAT proteins and their translocation to the nucleus, where dephosphorylated proteins show increased affinity for DNA $(18,19)$. Binding sites for NFAT proteins are present in the promoter/enhancer regions of several inducible genes, including the cytokines IL-2, IL-4, IL-5, IL-13, IFN- $\gamma$, and TNF- $\alpha$; the cell surface proteins CD40L, CTLA-4 and FasL; the enzyme cyclooxygenase-2, and the cell cycle regulator CDK4 $(1,4,15,20,21)$. Thus, a large number of inducible genes that regulate cell proliferation, differentiation, survival, and apoptosis are under the control of NFAT proteins.

\section{NFAT and cell cycle regulation}

The cell cycle is controlled by a set of protein complexes composed by two basic components: cyclin-dependent kinases (CDK) and their activation partners, the cyclins. Cyclin-CDK activity is regulated in response to appropriate stimuli and plays a key role in controlling cell cycle progression. The expression of specific cyclins is regulated at the transcription level, and dictates the formation of distinct cyclin/CDK complexes at different points of the cell cycle. CDK activity is usually influenced by several mechanisms, such as cyclin degradation (22) and phosphorylation of CDKs by inhibitory proteins, known as CDK inhibitors. CDK inhibitors are composed by two families of proteins: INK4 proteins, which specifically inhibit the catalytic subunit of CDK4 and CDK6, and Cip/Kip proteins, which affect the activity of cyclin D-, E-, and A-dependent kinases (23).

Different classes of cyclins are defined by the cell cycle phase at which they bind to CDKs and exert their function. During G0/ G1 transition phase, cyclin $\mathrm{C}$ stimulates CDK3-mediated phosphorylation of the retinoblastoma protein ( $\mathrm{pRB}$ ), exiting cells from quiescence (24). In G1 phase, cyclin DCDK4/6 complex phosphorylates pRB and leads to E2F transcription factor release, which transactivates genes related to $S$ phase entry $(23,25,26)$. During late G1, cyclin ECDK2 phosphorylates additional sites of the pRB subsequent to cyclin D-CDK4/6 phosphorylation sites, regulating G1/S transition. Cyclin A has been implicated in the control of S phase entry, as well as in the G2/M transition by binding to CDK2 and CDK1, respectively. Mitosis phase events are quite dependent on specific cyclin B-CDK1 complex activity $(23,26)$.

Increase of intracellular free calcium is central for cellular activation. Several observations have demonstrated that calcium signal stimulates gene transcription associated with cell cycle progression, and also promotes G1/S phase transition (27-29). Furthermore, accumulating evidence suggests that the phosphatase calcineurin plays a major role in the regulation of cell cycle progression by acting during the early stages of G1 phase $(20,30,31)$. Taken together, these results suggest that NFAT proteins, which are activated in consequence of calcium stimulus and calcineurin activity, are central regulators of the cell cycle machinery.

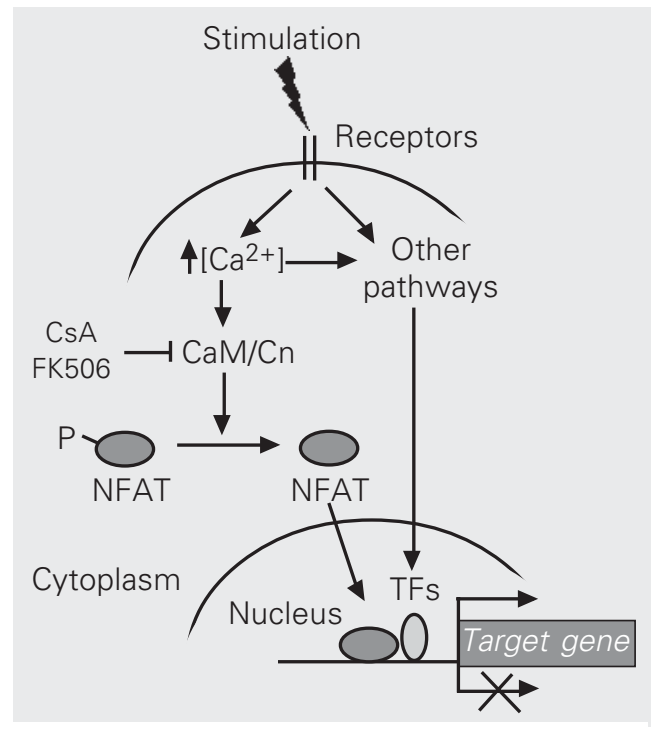

Figure 1. Schematic view of NFAT transcription factor signaling pathway in gene expression regulation. NFAT proteins are activated upon different stimuli that lead to increased intracellular calcium levels. When dephosphorylated by calcineurin, NFAT rapidly translocates to the nucleus where it regulates the expression of several target genes. NFATs may function as transcriptional activators or repressors and cooperate with several other transcription factors. $\mathrm{Ca}^{2+}=$ calcium; $\mathrm{CaM}=$ calmodulin; $\mathrm{Cn}=$ calcineurin; $\mathrm{CsA}$ = cyclosporin $\mathrm{A} ; \mathrm{NFAT}=$ nuclear factor of activated $T$ cells; $P=$ phosphorylation; TFs $=$ transcription factors. 
Recently, it has been demonstrated that the promoter of CDK 4 presents a consensus-binding site for NFAT proteins and is negatively regulated by NFAT1 without AP-1 cooperation (4). This finding demonstrates that NFAT1 directly controls the expression of CDK4 at the transcription level. Furthermore, NFAT1 has been implicated in the control of some cyclin genes, acting as a negative regulator of cyclins A2, E and B1 during lymphocyte activation (3). These results support the idea that the hyperproliferative phenotype observed in NFAT1-/- lymphocytes is related to a deregulated cell cycle progression, suggesting that NFAT1 transcription factor plays a central role in controlling cell cycle during $\mathrm{T}$ cell activation (3). Several other data also support this evidence. It has been shown that cyclosporin A and FK506 inhibit the expression of cyclins A and E in $3 \mathrm{~T} 3$ fibroblasts (31). Another evidence for the involvement of NFAT on cell growth has been shown in keratinocyte cells, where cyclosporin A suppressed the expression of $\mathrm{p} 21^{\mathrm{WAF} 1 / \mathrm{Cip} 1}$ and $\mathrm{p} 27^{\mathrm{KIP} 1}$, two CDK inhibitors (32). Moreover, transactivation of $\mathrm{p} 21$ promoter is upregulated by calcineurin and is dependent of Sp1/Sp3 in synergism with NFAT1 and NFAT2 (32). In addition, it has also been demonstrated that overexpression of NFAT2 in preadipocyte 3T3-L1 cells promoted cell cycle progression even under low serum concentrations and induced altered expression of cell cycle-related genes, such as cyclin D1, cyclin D2, pRB, and c-Myc (6). Finally, preliminary data from our laboratory suggest that NFAT1 directly regulates the expression of cyclin A2 gene (Carvalho LDS and Viola JPB, unpublished observations). This evidence supports the hypothesis that NFAT transcription factors control the expression of cell cycle-related genes and are central regulators of cell cycle progression.

\section{Cell cycle and $\mathrm{CD}^{+}{ }^{+} \mathrm{T}$ cell differentiation}

Upon primary stimulation, naive $\mathrm{CD} 4^{+} \mathrm{T}$ lymphocytes (T helper, Th) differentiate into two classes of effector cells with distinct functional abilities (33). During Th differentiation, these cells commit to a specific and mutually exclusive pattern of cytokine secretion (33). Th1 cells are characterized by the production of IFN- $\gamma$, contributing to cellular immune responses against intracellular pathogens, while Th2 cells produce IL-4, IL5 and IL-13, participating on the response against extracellular pathogens (33). Several factors can influence the differentiation pathway of Th cells, specially the conditions prevailing within the microenvironment where $T$ cells encounter antigen (34). However, the molecular basis for the cell-specific and mutually exclusive expression of Th1 or Th2 cytokines is not yet completely defined. Genetic experiments have implicated several transcription factors in Th cell differentiation, including NFAT, STAT4, STAT6, T-Bet and GATA-3. The proteins T-Bet and GATA-3 have been implicated as critical lineage-specific transcription factors which are sufficient to program Th1 and Th2 cytokine expression profiles, respectively $(35,36)$. Conversely, gene disruption of STAT4 and STAT6 indicated that these transcription factors also play major roles in Th differentiation $(37,38)$. However, in this section we will focus on reviewing data regarding the role of NFAT family proteins in Th differentiation.

NFAT proteins are present in both Th1 and Th2 cells, and can activate cytokine promoters in both cell types $(1,15)$. Also, NFAT transcriptional activity appeared to be similarly regulated during Th1/Th2 development, although it was enhanced in differentiated Th2 cells compared with differentiated Th1 cells (39). These similarities suggested that the activity of NFAT transcription factors were likely to overlap substantially. However, data generated by in vivo gene disruption of the different NFAT proteins suggest that they may have distinct roles in Th differentiation. NFAT1-/- mice 
developed normally, and did not exhibit any obvious behavioral deficiencies $(40,41)$. However, NFAT1-/- mice consistently showed a marked increase in their immune response. NFAT1-/- mice showed an enhanced $\mathrm{Th} 2$ development in both in vivo and in vitro models of Th differentiation, as evidenced by increased levels of IL-4 production $(40,42,43)$. Furthermore, cells from lymph nodes and spleen hyperproliferated in response to different antigen stimulations (41). Likewise, $\mathrm{CD} 4^{+} \mathrm{T}$ cells hyperproliferated in vitro in response to anti-CD3 (40). On the other hand, targeted disruption of NFAT2 resulted in an embryonic lethal phenotype $(9,15)$. Complementing RAG-1- or RAG-2deficient blastocysts with homozygous NFAT2-/- mutant ES cell lines demonstrated that $T$ and $B$ cells from chimeric mice showed reduced proliferation of peripheral lymphocytes when compared to wild-type mice $(44,45)$. In an in vitro model of Th differentiation, the chimeric mice displayed a decreased production of IL-4 but normal production of IFN- $\gamma$, demonstrating an impaired Th2 response in NFAT2-/- T cells $(44,45)$. Taken together, the results observed in NFAT-deficient mice demonstrated that these transcription factors play an important role in Th differentiation, and suggest that NFAT1 and NFAT2 have opposite roles in regulating gene expression in Th cell activation and differentiation. However, the molecular basis of these effects remains to be identified.

Cell cycle entry is one of the first events that occur during lymphocyte activation. Upon T cell receptor (TCR) activation, naive $\mathrm{T}$ lymphocytes, which are in a quiescent stage, proliferate, differentiate and acquire their effector functions. It has been shown that $\mathrm{T}$ cell differentiation is dependent on the cell cycle entry $(46,47)$. Although proliferation and differentiation of $\mathrm{CD}^{+}{ }^{+} \mathrm{T}$ cells occur concomitantly during cellular activation, it is still not clear whether they are effectively interconnected. Reiner and colleagues (46) have argued that cell cycle is an impor- tant regulator of Th differentiation. IL-4 and IFN- $\gamma$ production by naive $\mathrm{CD}^{+} \mathrm{T}$ cells could only be detected after one and three rounds of cellular division, respectively, being blocked by cell cycle-arresting drugs (46). Furthermore, it has been suggested that DNA synthesis is essential for the acquisition of IL-4 production competence in activated $\mathrm{CD} 4^{+} \mathrm{T}$ cells, since it is blocked by specific drugs that prevent cell cycle progression to $\mathrm{S}$ phase, but not to $\mathrm{G} 2 / \mathrm{M}$ phase (48). In contrast, it has been proposed that cell cycling only increases the frequency of cytokine-secreting cells, showing a correlation rather than dependence between cell cycle and differentiation $(47,49)$. In fact, non-cycling cells are capable of IFN- $\gamma$ secretion, suggesting that acquisition of competence to produce IFN- $\gamma$ can be achieved without entering into S phase (49). Other studies have argued that cell cycle progression is fundamental to the commitment of a Th cell with its cytokine-secreting profile, including the heritable activation/silencing of lineagedefining players (50). Actually, the reversibility of Th1 and Th2 populations is lost along the increasing number of cellular divisions and after long-term stimulation (47). Moreover, it has been shown that $\mathrm{T}$ cell differentiation is associated with a dynamic process of histone acetylation and chromatin remodeling at regulatory regions of IL-4 and IFN- $\gamma$ genes, suggesting that cell cycle could provide a window of opportunity to rearrange genes from an inactive to an active state $(47,51)$.

As discussed before, NFAT1-/- T cells show both hyperproliferation and increase in Th2 cytokine expression (40-42). However, it is still not clear whether the overexpression of Th2 cytokines, such as IL-4, is dependent on the hyperproliferative response observed in NFAT1-/- T cells or both phenomena are independent. We have hypothesized that NFAT transcription factors coordinately regulate the expression of earlyinducible genes that are involved in cell 
cycle control, such as cyclin genes, and in Th1/Th2 differentiation, such as IFN- $\gamma$ and IL-4 cytokines (3). It has been demonstrated that NFAT1 is able to bind to IFN- $\gamma$ promoter regions and to the IL-4 enhancer in T cells stimulated under Th1 or Th2 conditions, respectively (51). Since TCR stimulation activates an immediate and global chromatin derepression program in naive $\mathrm{T}$ cells, it has been suggested that TCR-inducible transcription factors, such as the preexisting cytoplasmic NFAT1 protein, may bind to regulatory regions of these cytokine genes, initiating localized chromatin remodeling (51). Finally, we indicate here that NFAT proteins regulate central events during lymphocyte activation, such as cell proliferation and cytokine expression, and may represent an informative model to elucidate the real correlation between cell cycle and Th differentiation.

\section{NFAT and malignant cell transformation}

As we discussed until here, NFAT proteins are involved in a variety of cellular mechanisms, and thus represent broader controllers of cell growth and development of normal cells. Several studies have already demonstrated definitive evidence for the involvement of NFAT proteins in the regulation of cell cycle progression, cell differentiation and cell death in different tissues $(3,6,52,53)$. These findings point to NFAT activation as an essential pathway in normal cell physiology, and therefore suggest that deregulation of NFAT signaling may occur during cellular aberrations and tumorigenic processes.

NFAT proteins have been shown to regulate cell cycle progression by modulating cyclin/CDK gene expression profile $(3,4)$. In mouse models, deficiency of NFAT1 transcription factor caused hyperproliferative cellular responses, altered cell cycle control, and increased stage-specific cyclin expres- sion in lymphocytes $(3,40,41)$. NFAT1 protein also repressed the CDK4 gene in T cells, dictating cell cycle re-entry from quiescence (4). Out of the immune system, NFAT1 deficiency also led to uncontrolled cell growth and differentiation of the connective tissue and skeletal muscle cells $(52,54)$. These results clearly implicate NFAT1 as a negative controller of early-inducible genes that regulate cell cycle progression and differentiation, and thus suggest a putative tumor suppressor role for this protein in different organ systems.

Although some members of the NFAT family are regulated in a similar way, they seem to play quite different effects during cellular responses. In contrast to NFAT1 effects, NFAT2 as well as NFAT3 and NFAT4 appear to function as inductors of cellular proliferation. NFAT2-deficient lymphocytes present a defect in $\mathrm{B}$ and $\mathrm{T}$ cell proliferation and an impaired repopulation of both thymus and peripheral lymphoid organs $(44,45)$. Recently, Neal and Clipstone (6) have shown that NFAT2 protein controls cellular proliferation of adipocytes by regulating cell cycle-related genes. In skeletal muscle and endothelial cells, NFAT2 activation was also essential for cellular growth and cardiac morphogenesis, respectively $(9,15,55)$. In accordance to NFAT2 data, several mouse models have elucidated the importance of NFAT3 and NFAT4 activation in smooth and skeletal muscle cell differentiation, indicating their role in blood vessels formation and angiogenesis $(16,29)$. These results provide evidence for a positive regulatory role of NFAT2 transcription factor in cell cycle machinery and growth signal autonomy, revealing its oncogenic potential.

Another hallmark of tumorigenesis is the ability that cancer cells present to evade cell death. Programmed cell death by apoptosis occurs in virtually all cell types and is precisely regulated at the cellular and molecular levels. Many molecular players have been characterized to take part in the apoptotic 
process, such as caspases, kinases and transcription factors. In fact, NFAT proteins have shown to bind to promoter regions and upregulate the expression of some effectors of apoptosis, such as TNF- $\alpha$ and FasL (1). It seems that NFAT1 presents a pro-apoptotic role in $\mathrm{T}$ lymphocytes since cells lacking this protein showed a decreased expression of FasL and TNF- $\alpha$ and were resistant to apoptosis (53). Moreover, overexpression of some NFAT members in primary $\mathrm{T}$ cells led to increased rates of apoptosis (53). These results thus suggest that NFAT deregulation may rescue normal cells from apoptosis and enable tumor cell survival. Conversely, some reports have also indicated anti-apoptotic roles for NFAT members. Overexpression of the NFAT2 protein in adipocytes protected cells from undergoing apoptosis in response to growth factor withdrawal (6). Mice lacking NFAT4 presented impaired expression of the anti-apoptotic protein Bcl2 and increased thymocyte apoptosis during $\mathrm{T}$ cell development in the thymus (56). Also, NFAT1 showed to rescue lymphocytes from activated-induced cell death upon antigen stimulation in vitro (3). In spite of the controversial data discussed, NFAT proteins are known regulators of apoptotic mechanisms and may take part in tumor development by sustaining cell survival.

Besides indirect evidence, there is plenty of data demonstrating the effective participation of NFAT proteins in tumor-related processes. Several in vitro and also in vivo reports support NFAT transcription factors as essential targets for better understanding tumor progression pathways. In an elegant work, Neal and Clipstone (6) have investigated the role of NFAT2 in tumorigenesis. A constitutively active form of the NFAT2 protein expressed in the murine 3T3-L1 preadipocyte cell line was able to induce cell transformation and inhibit cell differentiation. Upregulated NFAT2 activity induced the acquisition of well-defined hallmarks of tumorigenesis, including: 1) loss of contact- mediated growth inhibition, 2) reduced serum growth requirements, 3 ) protection from apoptosis, 4) formation of colonies in semisolid media, 5) acquisition of growth autonomy, and 6) formation of tumors in athymic nude mice (6). Moreover, authors speculate that sustained NFAT2 activity in vivo could be achieved by the continuous presence of a soluble promitogenic/prosurvival factor, which establishes an autocrine regulatory growth loop and leads to cell autonomy. These results provide strong evidence for the oncogenic potential of NFAT2 transcription factor and elicit its participation during tumor progression in vivo. Still, the expression of functional NFAT2 has been also identified in proliferating glioma cells, a nervous system-derived cell line. In line with this, cyclosporin A inhibited the proliferation of these cells and induced apoptotic cell death in a dose-dependent manner (57). Thus, the correlation between the presence of NFAT proteins and the proliferative status of glioma cells might converge to the participation of NFAT transcription factors in the transformed glioma cell phenotype by regulating an autocrine growth factor stimulation.

The formation of new blood vessels, a process called angiogenesis, is also essential in tumor development, since it supplies oxygen and nutrients during late stages of tumorigenesis. NFAT proteins have been implicated in the regulation of vascular endothelial growth factor-mediated angiogenesis by dictating the expression of cyclooxygenase2 , an enzyme that plays a pivotal role in neovascularization $(2,21)$. A very recent data also suggest NFAT1 protein as a convergent factor during angiogenesis, since it regulates the balance between stimulatory and inhibitory angiogenic signals (7). Beyond angiogenic processes, NFAT proteins have also been related to cellular metastasis. Rao and colleagues (58) have linked the integrinsignaling pathway to the activation of NFAT1 and NFAT5 proteins, which induced carcinoma invasion of cell lines derived from 
human breast and colon cancer. Integrins are extracellular matrix ligand receptors widely described as controllers of tumor invasion and migration. These results have highlighted NFAT proteins as relevant players in promoting tumor metastasis.

It is evident the involvement of NFAT transcription factors during tumorigenic processes (Figure 2). In accordance, antagonists of NFAT proteins inhibit tumor formation, supporting an oncogenic potential for these family of transcription factors (59). When binding to promoter regions and regulating gene expression, NFATs have shown to cooperate with several protooncogene products, such as c-Fos, c-Jun (AP-1), and Egr

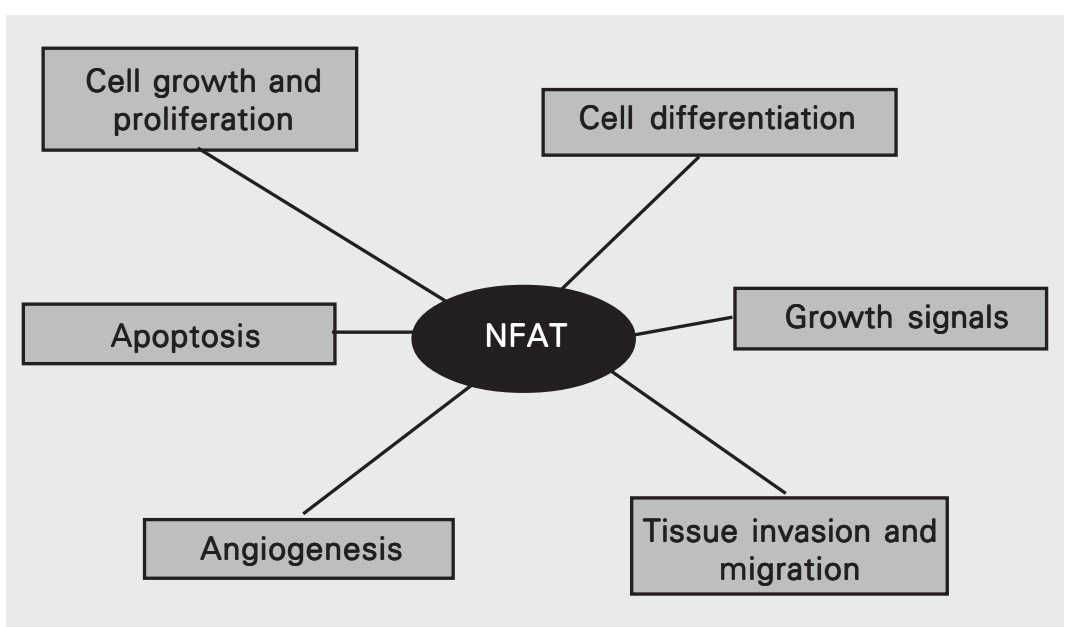

Figure 2. Tumor-related processes regulated by NFAT transcription factors. NFAT proteins have shown to directly regulate the expression of genes related to cellular proliferation (e.g., CDK4) and differentiation (e.g., cytokines and growth factors), apoptosis (e.g., FasL), angiogenesis (e.g., cyclooxygenase-2) and tissue invasion mechanisms among others. NFAT $=$ nuclear factor of activated $T$ cells. proteins $(1,60)$. These interactions may result in transcriptional activators or repressors in different moments (Figure 1). However, the subset of genes regulated by NFAT proteins and its importance during the different stages of tumor progression remain to be elucidated. Strong effort must focus on clarifying this still puzzling participation of NFATs during tumorigenesis.

\section{Final remarks}

NFAT transcription factors act as ubiquitous regulators of gene expression during cellular activation. NFAT proteins have diverse effects not only on cytokine expression, but also on cell cycle entry and apoptosis. Cumulative evidence has recently demonstrated that NFAT transcription factors exert important roles in the physiology of different cell types, and not only in cells related to the immune system. As such, NFAT has the potential to participate in malignant cell transformation related to cancer. As we discussed here, NFAT proteins regulate genes involved in cell cycle control, cell death and angiogenesis. Given the importance of these phenomena in the development of cell malignancies, it is of considerable interest to understand the mechanisms by which NFAT affects cell growth, differentiation and function. Finally, understanding more about the cell cycle regulation by NFAT proteins is central to precisely indicate the involvement of these transcription factors in malignant cell transformation and oncogenic processes.

\section{References}

1. Rao A, Luo C \& Hogan PG (1997). Transcription factors of the NFAT family: regulation and function. Annual Review of Immunology, 15: 707-747.

2. Hernández GL, Volpert OV, Íñiguez MA, Lorenzo E, MartínezMartínez S, Grau R, Fresno M \& Redondo JM (2001). Selective inhibition of vascular endothelial growth factor-mediated angiogenesis by cyclosporin A: roles of the nuclear factor of activated T cells and cyclooxygenase 2. Journal of Experimental Medicine, 193: 607620.
3. Caetano MS, Vieira-de-Abreu A, Teixeira LK, Werneck MBF, Barcinski MA \& Viola JPB (2002). NFATC2 transcription factor regulates cell cycle progression during lymphocyte activation: evidence of its involvement in the control of cyclin gene expression. FASEB Journal, 16: 1940-1942.

4. Baksh S, Widlund HR, Frazer-Abel AA, Du J, Fosmire S, Fisher DE, DeCaprio JA, Modiano JF \& Burakoff SJ (2002). NFATc2-mediated repression of cyclin-dependent kinase 4 expression. Molecular Cell, 10: 1071-1081. 
5. Graef IA, Wang F, Charron F, Chen L, Neilson J, Tessier-Lavigne M \& Crabtree GR (2003). Neurotrophins and netrins require calcineurin/NFAT signaling to stimulate outgrowth of embryonic axons. Cell, 113: 657-670.

6. Neal JW \& Clipstone NA (2003). A constitutively active NFATc1 mutant induces a transformed phenotype in 3T3-L1 fibroblasts. Journal of Biological Chemistry, 278: 17246-17254.

7. Zaichuk TA, Shroff EH, Emmanuel R, Filleur S, Nelius T \& Volpert $O$ (2004). Nuclear factor of activated T cells balances angiogenesis activation and inhibition. Journal of Experimental Medicine, 199: 1513-1522.

8. Shaw JP, Utz PJ, Durand DB, Toole JJ, Emmel EA \& Crabtree GR (1988). Identification of a putative regulator of early $T$ cell activation genes. Science, 241: 202-205.

9. Macian F, Lopez-Rodriguez C \& Rao A (2001). Partners in transcription: NFAT and AP-1. Oncogene, 20: 2476-2489.

10. McCaffrey PG, Luo C, Kerppola TK et al. (1993). Isolation of the cyclosporin-sensitive $\mathrm{T}$ cell transcription factor NFATp. Science, 262: $750-754$

11. Luo C, Burgeon E, Carew JA, Badalian TM, McCaffrey PG, Lane WS, Hogan PG \& Rao A (1996). Recombinant NFAT1 (NFATp) is regulated by calcineurin in $T$ cells and mediates the transcription of several cytokine genes. Molecular and Cellular Biology, 16: 39553966.

12. Northrop JP, Ho SN, Chen L, Thomas DJ, Timmerman LA, Nolan GP, Admon A \& Crabtree GR (1994). NF-AT components define a family of transcription factors targeted in T-cell activation. Nature, 369: 497-502.

13. Ho SN, Thomas DJ, Timmerman LA, Li X, Francke U \& Crabtree GR (1995). NFATc3, a lymphoid-specific NFATc family member that is calcium-regulated and exhibits distinct DNA binding specificity. Journal of Biological Chemistry, 270: 19898-19907.

14. Masuda E, Naito Y, Tokumitsu H, Campbell D, Saito F, Hannum C, Arai K-I \& Arai N (1995). NFATx, a novel member of the NFAT family that is expressed predominantly in the thymus. Molecular and Cellular Biology, 15: 2697-2706.

15. Crabtree GR \& Olson EN (2002). NFAT signaling: choreographing the social lives of cells. Cell, 109 (Suppl): S-67-S-79.

16. Horsley V \& Pavlath GK (2002). NFAT: ubiquitous regulator of cell differentiation and adaptation. Journal of Cell Biology, 156: 771-774.

17. Jain J, Burgeon E, Badalian TM, Hogan PG \& Rao A (1995). A similar DNA-binding motif in NFAT family proteins and the Rel homology region. Journal of Biological Chemistry, 270: 4138-4145.

18. Shaw KTY, Ho AM, Raghavan A, Kim J, Jain J, Park J, Sharma S, Rao A \& Hogan PG (1995). Immunosuppressive drugs prevent a rapid dephosphorylation of the transcription factor NFAT1 in stimulated immune cells. Proceedings of the National Academy of Sciences, USA, 92: 11205-11209.

19. Loh C, Shaw KTY, Carew J, Viola JPB, Luo C, Perrino BA \& Rao A (1996). Calcineurin binds the transcription factor NFAT1 and reversibly regulates its activity. Journal of Biological Chemistry, 271: 10884-10891.

20. Baksh S, DeCaprio JA \& Burakoff SJ (2000). Calcineurin regulation of the mammalian G0/G1 checkpoint element, cyclin dependent kinase. Oncogene, 19: 2820-2827.

21. Iniguez MA, Martinez-Martinez S, Punzon C, Redondo JM \& Fresno $M$ (2000). An essential role of the nuclear factor of activated T cells in the regulation of the expression of the cyclooxygenase-2 gene in human T lymphocytes. Journal of Biological Chemistry, 275: $23627-$ 23635.

22. Koepp DM, Harper JW \& Elledge SJ (1999). How the cyclin became a cyclin: regulated proteolysis in the cell cycle. Cell, 97: 431-434.

23. Sherr CJ \& Roberts JM (1999). CDK inhibitors: positive and negative regulators of G1-phase progression. Genes and Development, 13: 1501-1512.

24. Ren S \& Rollins BJ (2004). Cyclin C/Cdk3 promotes Rb-dependent G0 exit. Cell, 117: 239-251.

25. Ohtani K, DeGregori J \& Nevins JR (1995). Regulation of the cyclin E gene by transcription factor E2F1. Proceedings of the National Academy of Sciences, USA, 92: 12146-12150.

26. Murray AW (2004). Recycling the cell cycle: cyclins revisited. Cell, 116: $221-234$

27. Anraku Y, Ohya $Y$ \& lida H (1991). Cell cycle control by calcium and calmodulin in Saccharomyces cerevisiae. Biochimica et Biophysica Acta, 1093: 169-177.

28. Tomono M, Toyoshima K, Ito M \& Amano H (1996). Calcineurin is essential for DNA synthesis in Swiss 3T3 fibroblasts. Biochemical Journal, 317: 675-680.

29. Lipskaia L \& Lompré AM (2004). Alteration in temporal kinetics of $\mathrm{Ca}^{2+}$ signaling and control of growth and proliferation. Biology of the Cell, 96: 55-68.

30. Terada N, Lucas JJ \& Gelfand EW (1991). Differential regulation of the tumor suppressor molecules, retinoblastoma susceptibility gene product $(\mathrm{Rb})$ and $\mathrm{p53}$, during cell cycle progression of normal human T cells. Journal of Immunology, 147: 698-704.

31. Tomono M, Toyoshima K, Ito M, Amano H \& Kiss Z (1998). Inhibitors of calcineurin block expression of cyclins $A$ and $E$ induced by fibroblast growth factor in Swiss 3T3 fibroblasts. Archives of Biochemistry and Biophysics, 353: 374-378.

32. Santini MP, Talora C, Seki T, Bolgan L \& Dotto GP (2001). Cross talk among calcineurin Sp1/Sp3 and NFAT in control of p21WAF1/CIP1 expression in keratinocyte differentiation. Proceedings of the $\mathrm{Na}$ tional Academy of Sciences, USA, 98: 9575-9580.

33. Abbas AK, Murphy KM \& Sher A (1996). Functional diversity of helper T lymphocytes. Nature, 383: 787-793.

34. Constant SL \& Bottomly K (1997). Induction of Th1 and Th2 CD4+ T cells response: the alternative approaches. Annual Review of Immunology, 15: 297-322.

35. Zheng W \& Flavell RA (1997). The transcription factor GATA-3 is necessary and sufficient for Th2 cytokine gene expression in CD4 T cells. Cell, 89: 587-596.

36. Szabo SJ, Kim ST, Costa GL, Zhang X, Fathman CG \& Glimcher LH (2000). A novel transcription factor T-bet directs Th1 lineage commitment. Cell, 100: 655-669.

37. Kaplan MH, Schindler U, Smiley ST \& Grusby MJ (1996). Stat6 is required for mediating responses to IL-4 and for development of Th2 cells. Immunity, 4: 313-319

38. Kaplan MH, Sun YL, Hoey T \& Grusby MJ (1996). Impaired IL-12 responses and enhanced development of Th2 cells in Stat4-deficient mice. Nature, 382: 174-177.

39. Rincon M \& Flavell RA (1997). T-cell subsets: transcriptional control in the Th1/Th2 decision. Current Biology, 7: R729-R732.

40. Hodge MR, Ranger AM, Charles de la Brousse F, Hoey T, Grusby MJ \& Glimcher LH (1996). Hyperproliferation and dysregulation of IL-4 expression in NF-ATp-deficient mice. Immunity, 4: 397-405.

41. Xanthoudakis S, Viola JP, Shaw KT, Luo C, Wallace JD, Bozza PT, Luk DC, Curran T \& Rao A (1996). An enhanced immune response in mice lacking the transcription factor NFAT1. Science, 272: 892-895.

42. Kiani A, Viola JPB, Lichtman AH \& Rao A (1997). Downregulation of IL-4 gene transcription and control of Th2 cell differentiation by a mechanism involving the transcription factor NFAT1. Immunity, 7: 849-860. 
43. Viola JPB, Kiani A, Bozza PT \& Rao A (1998). Regulation of allergic inflammation and eosinophil recruitment in mice lacking the transcription factor NFAT1: Role of interleukin-4 (IL-4) and IL-5. Blood, 91: 2223-2230.

44. Ranger AM, Hodge MR, Gravallese EM, Oukka M, Davidson L, Alt FW, Charles de la Brousse F, Hoey T, Grusby M \& Glimcher LH (1998). Delayed lymphoid repopulation with defects in IL-4-driven responses produced by inactivation of NF-ATc. Immunity, 8: 125134.

45. Yoshida H, Nishina H, Takimoto $H$ et al. (1998). The transcription factor NF-ATc1 regulates lymphocyte proliferation and Th2 cytokine production. Immunity, 8: 115-124.

46. Bird JJ, Brown DR, Mullen AC, Moskowitz NH, Mahowald MA, Sider JR, Gajewski TF, Wang C-R \& Reiner SL (1998). Helper T cell differentiation is controlled by the cell cycle. Immunity, 9: 229-237.

47. Grogan JL, Mohrs M, Harmon B, Lacy DA, Sedat JW \& Locksley RM (2001). Early transcription and silencing of cytokine genes underlie polarization of T helper cell subsets. Immunity, 14: 205-215.

48. Richter A, Löhning $M$ \& Radbruch A (1999). Instruction for cytokine expression in $\mathrm{T}$ helper lymphocytes in relation to proliferation and cell cycle progression. Journal of Experimental Medicine, 190: 14391450.

49. Ben-Sasson SZ, Gerstel R, Hu-Li J \& Paul WE (2001). Cell division is not a "clock" measuring acquisition of competence to produce IFN$\gamma$ or IL-4. Journal of Immunology, 166: 112-120.

50. Mullen AC, Hutchins AS, Villarino AV, Lee HW, High FA, Cereb N, Yang SY, Hua X \& Reiner SL (2001). Cell cycle controlling the silencing and function of mammalian activators. Current Biology, 11: 1695-1699.

51. Avni O, Lee D, Macian F, Szabo S, Glimcher L \& Rao A (2002). Th cell differentiation is accompanied by dynamic changes in histone acetylation of cytokine genes. Nature Immunology, 3: 643-651.

52. Ranger AM, Gerstenfeld LC, Wang J, Kon T, Bae H, Gravallese EM, Glimcher MJ \& Glimcher LH (2000). The nuclear factor of activated T cells (NFAT) transcription factor NFATp (NFATc2) is a repressor of chondrogenesis. Journal of Experimental Medicine 191: 9-22.

53. Chuvpilo S, Jankevics E, Tyrsin D et al. (2002). Autoregulation of NFATc1/A expression facilitates effector $T$ cells to escape from rapid apoptosis. Immunity, 16: 881-895.

54. Horsley V \& Pavlath GK (2003). Prostaglandin F2 $\alpha$ stimulates growth of skeletal muscle cells via an NFATC2-dependent pathway. Journal of Cell Biology, 161: 111-118.

55. Musaro A, McCullagh KJ, Naya FJ, Olson EN \& Rosenthal N (1999). IGF-1 induces skeletal myocyte hypertrophy through calcineurin in association with GATA-2 and NF-ATc1. Nature, 400: 581-585.

56. Oukka M, Ho IC, De la Brousse FC, Hoey T, Grusby MJ \& Glimcher LH (1998). The transcription factor NFAT4 is involved in the generation and survival of T cells. Immunity, 9: 295-304.

57. Mosieniak G, Figiel I \& Kaminska B (1997). Cyclosporin A, an immunosuppressive drug, induces programmed cell death in rat C6 glioma cells by a mechanism that involves the AP-1 transcription factor. Journal of Neurochemistry, 68: 1142-1149.

58. Jauliac S, López-Rodriguez C, Shaw LM, Brown LF, Rao A \& Toker A (2002). The role of NFAT transcription factor in integrin-mediated carcinoma invasion. Nature Cell Biology, 4: 540-544.

59. Jiang H, Yamamoto S, Nishikawa K \& Kato R (1993). Anti-tumorpromoting action of FK506 a potent immunosuppressive agent. Carcinogenesis, 14: 67-71.

60. Rengarajan J, Mittelstadt PR, Mages HW, Gerth AJ, Kroczek RA Ashwell JD \& Glimcher LH (2000). Sequential involvement of NFAT and Egr transcription factors in FasL regulation. Immunity, 12: 293300 . 